\title{
[ARTIGO RETRATADO] Qualidade de vida de cuidadores de idosos vinculados ao Programa Saúde da Família - Teixeiras, MG
}

\section{Quality of life of elderly caregivers of link to the Family Health Program - Teixeiras, MG}

\begin{abstract}
Paulo Henrique Bittencourt Moreira'; Simone Caldas Tavares Mafra ${ }^{\mathrm{II}}$; Eveline Torres Pereira ${ }^{\mathrm{III}}$; Vania Eugênia da Silva ${ }^{\mathrm{I}}$

IPrograma de Pós-Graduação em Economia Doméstica da Universidade Federal de Viçosa. Viçosa, MG, Brasil.

II Departamento de Economia Doméstica da Universidade Federal de Viçosa. Viçosa, MG, Brasil.

IIIDepartamento de Educação Física da Universidade Federal de Viçosa. Viçosa, MG, Brasil.
\end{abstract}

A Revista Brasileira de Geriatria e Gerontologia (RBGG) solicita que seja retirado do sistema SciELO o artigo Qualidade de vida de cuidadores de idosos vinculados ao Programa Saúde da Família - Teixeiras, MG (Quality of life of elderly caregivers of link to the Family Health Program - Teixeiras, MG) de Paulo Henrique Bittencourt Moreira; Simone Caldas Tavares Mafra; Eveline Torres Pereira e Vania Eugênia da Silva, publicado no volume 14, no 3, julho-setembro/2011, págs.433-440, por motivo de plágio. Diversos parágrafos foram transcritos fielmente do artigo previamente publicado pelos professores AMENDOLA, Fernanda; OLIVEIRA, Maria Amélia de Campos e ALVARENGA, Márcia Regina Martins. Os autores da fraude acadêmica admitem a falha ética e apresentam suas sinceras desculpas. Abaixo a fonte original:

1. AMENDOLA, Fernanda; OLIVEIRA, Maria Amélia de Campos e ALVARENGA, Márcia Regina Martins. Qualidade de vida dos cuidadores de pacientes dependentes no programa de saúde da família. Texto contexto - enferm. [online]. 2008, vol.17, n.2 [cited 2013-03-05], pp. 266-272 . Available from: <http://www.scielo.br/scielo.php?script=sci_arttext\&pid=S0104-07072008000200007\&lng=en\&nrm=iso>. ISSN 0104-0707. http://dx.doi.org/10.1590/S0104-07072008000200007.

A fraude acadêmica foi informada pela Profa.Dra. Márcia Regina Martins Alvarenga, uma das autoras do artigo plagiado. Assim, a Comissão Editorial julgou por bem solicitar a retirada do artigo do sistema SciELO.

Agradecemos sua atenção e providências.

Prof. Dr. Renato Veras

Editor da RBGG 


\section{Qualidade de vida de cuidadores de idosos vinculados ao Programa Saúde da Família - Teixeiras, MG}

Quality of life of elderly caregivers of link to the Family Health Program - Teixeiras, MG

Resumo

O estudo teve como objetivo avaliar a associação entre a percep $\mu$ su jetiva la qualidade de vida dos cuidadores domiciliares de idosos vincula 'os ar pro ama Saúde da Família (PSF) e as características sociodemogr' cas e c saúde, grau de sobrecarga percebida e grau de independência fu 10 , 1 do ido 5 . Foram entrevistados 66 cuidadores, de agosto a dezem ${ }^{1}$ o de 20 \&, utilizando os instrumentos WHOQOL-bref; Zarit Burden Interv e a Medida da independência Funcional para os idosos. Houve o predomínic te $\mathrm{r}$ theres cuidadoras, donas de casa e em média com 50,5 anos. A média ' nedic de in -pendência funcional foi 57,82 , classificando a amostra de ido s em enena cra modificada. A escala Zarit correlacionou-se negativamente $\mathrm{A}$ od os a mínios do WHOQOL-bref. As variáveis independentes estatisticame e relacionadas à qualidade de vida geral, no modelo múltiplo companheiro e a presença ${ }^{\prime}$ doença o cuidador, considerando, em especial, o fato de que o baixo nível d escolarida e e de renda pode afetar a forma como o cuidado é realizado.

\section{Abstract}

The $s, a$, aimed to yaluate the association between the subjective perception of auality o. 'ife of caregivers of elderly households linked to the Family Health rog $\mathrm{m}(\mathrm{FHr}$, and the socio-demographic characteristics and health, level of perc $m$ burden and degree of functional independence of the elderly. From

Pr grama de Pós-Graduação em Economia Doméstica da Universidade Federal de Viçosa. Viçosa, MG, Brasil.

2 Departamento de Economia Doméstica da Universidade Federal de Viçosa. Viçosa, MG, Brasil. Departamento de Educação Física da Universidade Federal de Viçosa. Viçosa, MG, Brasil.

Artigo extraído da dissertação de mestrado intitulada "Análise da sobrecarga de trabalho e da qualidade de vida dos cuidadores domiciliares de idosos”, defendida no Programa de Pós-Graduação em Economia Doméstica, da Universidade Federal de Viçosa, MG, em 2009.

Correspondência / Correspondence

Simone Caldas Tavares Mafra

E-mail:sctmafra@ufv.br
Palavras-chave: Qualidade de Vida. Cuidador. Saúde da família. Serviços de Saúde. Idoso de 80 anos ou mais. Saúde do Idoso. 
August 2008 to December 2008, 66 caregivers were interviewed, using the instruments WHOQOL-bref, Zarit Burden Interview and the Functional Independence Measure for the elderly. There was predominance of female caregivers, housewives and, on average, aged 50.5 years. The average measure of functional independence was 57.82 classifying a sample of elderly dependency on modified. The Zarit scale correlated negatively with all domains of WHOQOL-bref. The independent variables statistically related to overall quality of life in multiple models were: the overload scale, the presence of companion and the presence of disease in caregivers, especially considering the fact that the bass of education level and of income they can affect the way care is accomplished.
Key words: Quality of Life. Caregivers. Family Health. Services of Health. Elderly of 80 years or more. Health of the elderly one.

\section{INTRODUÇÃO}

As mudanças nos perfis demográficos e de morbi-mortalidade da população mundial ao longo do último século e consequentes desafios para os sistemas de saúde explicam o crescente interesse dos pesquisadores pelo cuidado domiciliário à saúde, realizado no âmbito da família e envolvendo cuidadores leigos. Com a implementação do Programa Saúde da Famí1 ' (PSF) na Atenção Básica (AB), os cuida os domiciliários de saúde tornaram- a arto integrante das ações em saúde ne c nívf de atenção. O cadastramento das famílias i pelos agentes comunitários de saúde levou- aos domicílios de usuários corm y visíveis as necessidades de saúde de $\mathrm{i}$ osos, ant confinados em seus lares, e de senc cur 'adores. ${ }^{1}$

Estes, por sua ez, oc pai napel especial, já que são eles qu di urnam nte participam da

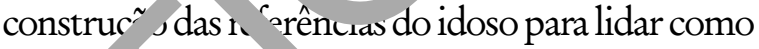
seu ce a 'o. Suas tu fas muitas vezes são atribuídas sem zorient a $_{\text {. }}$ o adequada, o suporte das instituições de $s$ áde, a arteração das rotinas, e o tempo der ena $J$ no cuidado tem impacto sobre a q. lidade de vida do cuidador. É de se esperar que quaidade de vida do cuidador domiciliar principal seja afetada negativamente por tais circunstâncias, o que é corroborado por vários estudos. ${ }^{1-3}$ Ainda assim, qualidade de vida e sobrecarga são construtos diferentes, e que, portanto, devem ser abordados com diferentes instrumentos de medida.

A qualidade de vida tornou-se um conceito amplamente difundido em diversas áreas, sobretudo no meio ? têmico, or meio de estudos científicos relaciona 'os à saude. ${ }^{4-6}$ Alguns desses estudos têr cido critican s por autores que alegam que os ins un tos criados para medir a qualidade vida verdade avaliam diferentes fenôm as. ${ }^{7} \mathrm{Is}$ a dec re da falta de consenso sobre o cr ast to daco. dplexidade do fenômeno que pretend valiar. Há, contudo, concordância sobı fato de que a qualidade de vida é composta por asp tos objetivos e subjetivos, positivos e negativos. As avaliações objetivas referem-se às cc sequências observáveis, enquanto as subjetivas dizem respeito à percepção ou avaliação pessoal sobre determinado aspecto abordado.

Nesta investigação, optou-se por utilizar o questionário de avaliação subjetiva de Qualidade de Vida (QV) desenvolvido pela Organização Mundial da Saúde (OMS), o World Health Organization Quality of Life instrument-bref (WHOQOL-bref), versão abreviada do World Health Organization Quality of Life instrument-100 itens (WHOQOL-100). Trata-se de um instrumento que adota um conceito multidimensional de QV, é amplamente utilizado no Brasil e em outros países e foi construído por meio de um levantamento sobre o que as pessoas consideram importante abordar ao medir QV. Este instrumento teve grande importância para atender ao objetivo do estudo, que foi analisar a QV do cuidador domiciliar, pelo fato de este estudo ter como pressuposto que o cuidador domiciliar vivencia a situação de redução da sua QV em função de não dispor de rede de apoio para esta atividade. Nesse sentido, a pesquisa apresentada se justificou, considerando, em especial, que 
buscou descrever e compreender o cotidiano do cuidado domiciliar do idoso. ${ }^{4}$

A avaliação da qualidade de vida dos cuidadores domiciliares deve contemplar tanto os aspectos positivos quanto os negativos, pois muitas situações consideradas negativas por meio de indicadores objetivos podem ser subjetivamente percebidas como positivas pelos indivíduos em questão. Tanto a dimensão objetiva (exemplificada por questões relacionadas à saúde do indivíduo, ou seja, apresentar ou não alterações em sua saúde) quanto a subjetiva (que pode ser exemplificada pela existência ou não de redes de apoio, e que no caso da ausência destas, pode deflagrar problemas de saúde no cuidador), é importante para o enriquecimento das informações coletadas com a finalidade de avaliar a qualidade de vida de cuidadores, permitindo melhor interpretar sua realidade de vida e saúde.

O presente estudo buscou analisar a associação entre a percepção subjetiva da qualidade de vida de cuidadores domiciliares de idosos vinculad is ao PSF do município de Teixeiras - MG, s características sociodemográficas; a cor $\mathrm{coc}^{+}$, de saúde dos cuidadores; as característi is do ; (idade, sexo, diagnóstico e dependência l cional) e o escore da sobrecarga abordado a partir a. MIF.

\section{METODOLOGIA}

Trata-se de es do descr trvo e transversal, no qual foran $\mathrm{r}$ liza atravé te ques nnário estruturado e escalas padroniza ' ' 5 , ,8,9 apricadas a cuidadores e idosos Ail lados ac Programa de Saúde da Família, da cida Te Teixeiras - MG, no período de agosto/ $\Delta 8$ a dezembro/2008.

$O$ ano de referência para implantação do PSF no município de Teixeiras é 1997 , sendo a cobertura total da população alcançada em 1999. Atualmente, estão em funcionamento quatro equipes de saúde da família, compostas por 24 microáreas de saúde.

A amostra foi composta por 66 cuidadores domiciliares de idosos, atendida por equipes de
Saúde da Família distribuídas entre quatro unidades básicas de saúde. Foram enviadas cartasconvite às quatro unidades para que participassem do estudo, e todas responderam à solicitação. Foram identificados 95 cuidadores, dos quais foram excluídos dez que não se encaixavam no critérios de seleção. Dentre os 85 possív is participantes, houve uma recusa, do = falecimentos e três mudanças de $\mathrm{r} / \mathrm{nICl}_{\mathrm{P}}$ ?. Outras 13 entrevistas não puderam st realizada porque o cuidador não foi enc $n$ trado $n c$ ? ou por incompatibilidade de ho ' $\mathrm{rlo}$, $\mathrm{m}$ a culeta de dados, totalizando, as in, 66 adores.

Os critérios de inclus para seleção dos sujeitos foram: dadores co, idade igual ou maior de 16 an ss, unerados ou não e prestandr _ dado há mais de dois meses ao idoso empo ansi erado mínimo no que se ref e a arecimento da doença, ou seja, o rimento la necessidade de cuidado junto ao idos No entanto, a referida pesquisa evidenciou cuidado as que tiveram tempo mínimo de três meses a 42 anos (ou seja, as esposas cuidando de sf ss maridos), evidenciando uma média de cuidado próxima de 9,8 anos. Foram excluídas as famílias na qual não foi possível identificar o cuidador principal. Definiu-se cuidador a pessoa que assume o papel de assistir ao idoso em situação de dependência ou não e também que seja remunerado ou não.

O grau de dependência do idoso foi determinado pela Medida de Independência Funcional (MIF), ${ }^{8}$ que também verificou sua reprodutibilidade. O instrumento é composto por 18 categorias agrupadas em seis dimensões: autocuidado, controle de esfíncteres, transferências, locomoção, comunicação e cognição social. Cada item pontua de 1 (dependência total) a 7 (independência completa). Obtém-se escore total mínimo de 18 e máximo de 126 pontos que caracterizam os níveis de dependência.

Para mensurar a sobrecarga dos cuidadores, utilizou-se a escala Zarit Burden Interview (ZBI), composta por 22 itens que avaliam a relação cuidador-paciente, a condição de saúde, o bem- 
estar psicológico, finanças e vida social. A escala de respostas varia de $\mathrm{O}$ a 4 , de acordo com presença ou intensidade de uma resposta afirmativa $(0=$ nunca, $1=$ raramente, $2=$ algumas vezes, 3 =frequentemente e $4=$ sempre). A exceção é o último item, no qual o entrevistado é questionado se está se sentindo sobrecarregado no papel de cuidador e as respostas são: $0=$ nem um pouco, $1=$ um pouco, $2=$ moderadamente, $3=$ muito, $4=$ extremamente. $O$ escore total da escala varia de 0 a 88. Quanto maior o escore, maior a sobrecarga.

O questionário utilizado para avaliar a QV dos cuidadores, o WHOQOL-bref, ${ }^{4}$ contém 26 questões, sendo duas gerais (qualidade de vida geral e satisfação com a saúde) e 24 englobadas nos domínios Físico, Psicológico, Relações Sociais e Meio Ambiente. As duas questões gerais são calculadas em conjunto para gerar um único escore independente dos outros escores dos domínios, no qual denominamos de Índice Geral de Qualidade de Vida (IGQV).

O tratamento dos dados foi feito utiliz $\cdots$ do-s. o softwrare Statistical Packege for the Social s, ences 20. Para avaliar a correlação das variáveis qu nt' ativa do cuidador e do paciente com a QV do cu Jador, utilizou-se o coeficiente de c ncm õo de Spearman. Também foi estimado ur modelo le regressão linear múltipla, tendo con variáve dependente o escore do IGQV como var m uldependentes, as características : sciod no áficas, a condição de saúde dos cur ' ' do. 's, as car cterísticas do paciente (idade, $, \mathrm{s}, \mathrm{O}$, dia $a_{\varepsilon}$ 'óstıue dependência funcional - MIF, e escore du obrecarga. Para este estudo, eloreu-se ap as o escore do IGQV, por considerá1o ur indicador mais abrangente e adequado aos ob ivo do estudo.

1) Jram selecionadas para o processo de nıodelagem múltipla aquelas variáveis com $\mathrm{p}<0,20$. O processo de seleção foi o stepwrise forvard e a variável independente permaneceu no modelo múltiplo quando $\mathrm{p}<0,05$. O nível de significância adotado para o estudo foi de $5 \%$.

Obteve-se aprovação do Programa de PósGraduação em Economia Doméstica/UFV, do
Comitê de Ética em Pesquisa da Secretaria Municipal de Saúde de Teixeiras (nº 041-2008), e da coordenação do PSF do município. Os participantes, depois de informados sobre os objetivos da pesquisa, assinaram o Termo de Consentimento Livre e Esclarecido.

\section{RESULTADOS}

Os cuidadores eram, em sua maioria, in ther s $(83,3 \%)$, filhas $(37,9 \%)$, casaa 50 . คm u..iâo consensual $(62,2 \%)$ com tédia c ade de 50,5 anos $(\mathrm{dp}=14,84$; med; $\mathrm{M}$. de 51 an $)$ e referiram ter alguma doença $(63,6 \%)$.

Entre os cuida res dominou o diagnóstico de hiper ão a crial $(39,4 \%)$ e depressão $\left(13,6^{\circ}\right.$, , ma nó $\mathrm{h}$ )uve registro de uso de me "cau os paru depressão ou calmantes, não rendo $r$ to do uso de medicamentos para hipt ensão, embora um grande número de cuidado es tenha apresentado o diagnóstico. Acredita-se que o uso do calmante seja em função dr estresse que a atividade envolve e para redução do desconforto da dor, visto que $54,5 \%$ referiram algum tipo de dor e dormiam em média sete horas por dia.

O tempo como cuidador variou de três meses a 42 anos, com uma média de 9,8 anos cuidando do idoso ( $\mathrm{dp}=9,6$ anos) e mediana de seis anos.

A escala de avaliação da sobrecarga $Z B I$ variou de 0 a 67, com média de 32,12 ( $\mathrm{dp}=14,7)$. O alpha de Cronbach para o ZBI foi de 0,86, demonstrando bom índice de consistência interna para esta amostra.

No WHOQOL-bref, os domínios Físico e Relações Sociais apresentaram os maiores escores (66,72 e 60,86, respectivamente), enquanto que o domínio Meio Ambiente apresentou o menor $(52,51)$. A confiabilidade do instrumento, avaliada pelo alpha de Cronbach, mostrou bom índice de consistência interna nos domínios Físico e Psicológico ( $0,82 \mathrm{e} 0,74$, respectivamente) e baixo nos domínios Relações Sociais e Meio Ambiente $(0,49 \mathrm{e} 0,45$, respectivamente). 
Em relação à correlação da $\mathrm{QV}$ do cuidador com as variáveis quantitativas do cuidador e do idoso, através da correlação de Spearman, destacase a correlação negativa estatisticamente significativa no domínio Físico com a idade do cuidador e a idade do idoso e no IGQV e todos os demais domínios com a escala Zarit, indicando que quanto menor o escore médio do IGQV e dos demais domínios, maior o escore médio do Zarit - portanto, quanto maior a sobrecarga, menor os escores de QV.

A maioria dos idosos era do sexo feminino $(57,6 \%)$ e com média de 63,45 anos $(\mathrm{dp}=22,5)$. A patologia dos pacientes mais citada pelos cuidadores foi acidente vascular encefálico $(39,4 \%)$. A média total da escala MIF foi de 57,82 $(\mathrm{dp}=30,28)$ e a mediana de 53,0 , classificando a amostra em dependência modificada. As pontuações mais baixas foram encontradas nos domínios autocuidado $(17,45)$, transferência $(8,73)$ e locomoção $(4,76)$. O valor de alpha de Cronbach de cada domínio e da escala total foi bastan satisfatório em todos os domínios (acima de 0, ?). Acredita-se que isso ocorra pelo fato $\mathrm{d} \rho$ tosc ser do sexo feminino, e neste aspecto liter $\cdots$ a menciona que o cuidador sente como in "al este cuidado, considerando que a pessoa cuic ta ao longo de sua vida oferecev culda ? merecendo neste momento de sua vic toda a at nção do seu cuidador. Neste aspecto, ão é pe cebido pelo cuidador o estres $i$ do atr de cuudr, mesmo que este exista, mas ، prazur do "to de se "doar".

As $v$ ráveis depcridentes estatisticamente relac' $\mathrm{n}_{a}$ 'as com o GQV foram a escala de Zarit $(b--0,542,>=0,001)$, ter companheiro(a) $(b=$ +10. $55 ; \mathrm{p}=0,039)$ e a presença de doença no cu ado, $b=-11,701 ; p=0,021)$. Conclui-se que p. a cada ponto do Zarit, há, em média, uma dimınuição de 0,542 no IGQV. O cuidador casado ou em união consensual (com companheiro) tem em média mais 10,355 pontos no IGQV. O cuidador que apresenta alguma doença tem em média menos 11,701 pontos no IGQV, quando comparado àquele que não refere doenças. Feita a análise de resíduos, verificou-se que, do ponto de vista estatístico, o modelo final não apresenta viés.

\section{DISCUSSÃO}

A QV foi eleita como a variável dependente do estudo e os outros dados como variáveis independentes, buscando correlacionar as variáveis exploradas tanto relativas aos cuidadores quant aos pacientes com o grau de implicação na Q

Os cuidadores domiciliares, obi to a te estudo, foram selecionados não en função c doença do idoso, mas pela insercão de an ' hos e. 1 um determinado nível de atenç 'O $\mathrm{c}$. cisteı, a de saúde, como é o caso d Prog "ra Saude da Família, que integra enção b sica. Poucos estudos avaliam os cuida 'ores ha Atenção Básica, ${ }^{10,11}$ sendo - nonderante os que avaliam o cuidador em funç, od $\leadsto$ nça do idoso ${ }^{6,11}$ ou por serviços y lados hospitais. ${ }^{1,2}$

- ma a dos cosos era do sexo feminino. A dia de idos atendidos pelas equipes de saúde da família, em gera conseguem realizar as tarefas básicas $\mathrm{da}$ vida diária em até $50 \%$. Ou seja, a maioria dos p rentes apresenta necessidades de cuidado, mas essas pessoas não conseguem realizar algumas atividades da vida diária sem a ajuda de um cuidador. Acredita-se que o fato de existir uma dependência moderada, como mencionado anteriormente, não afete sobremaneira a QV do cuidador no que se refere aos dados investigados pela MIF, como descrito na metodologia do estudo.

O predomínio de mulheres cuidadoras, casadas, donas de casa e em média com 50,5 anos, também são características frequentes encontradas em diversos estudos, seja no âmbito nacional ou internacional. ${ }^{1,12-14}$

Os achados reforçam o papel social da mulher, historicamente determinado, onde é esperado que ela seja a cuidadora principal e que o cuidado a pessoas idosas fique a cargo dos parentes mais próximos. A escolaridade é predominantemente baixa em diversos estudos com cuidadores. ${ }^{1,3,11} \mathrm{O}$ baixo nível de escolaridade pode contribuir para o papel de cuidador ser atribuído a essas pessoas, na medida em que se sabe que a inserção no 
mercado de trabalho formal é mais difícil para os indivíduos com baixa escolaridade. Assim, é mais provável que essas pessoas se dediquem aos serviços domésticos e à tarefa de cuidar do idoso como uma extensão dessa atividade.

$\mathrm{O}$ índice médio de sobrecarga do cuidador, avaliado através do ZBI, foi de 32,12 pontos portanto, estavam moderadamente sobrecarregados. Estudos com cuidadores de idosos que utilizaram essa escala apresentaram dados semelhantes, indicando escores de moderado a alto. ${ }^{2,15}$

A idade do idoso mostrou correlação com o domínio Físico, tanto na escala de Zarit, quanto na avaliação da MIF, indicando que quanto maior a idade, menor o escore médio nesse domínio, já que idosos mais velhos requerem cuidados que sobrecarregam fisicamente $o$ cuidador. A idade do idoso também se correlacionou com o domínio Meio Ambiente, considerando o que prevê o WHOQOL-bre, ou seja, quanto maior a idade, menor o es, re do domínio. O idoso normalmente demana. condições específicas para seu cuidad , devj lo à diminuição de algumas capacidades au ${ }^{\circ}$ ro $\mathrm{o}$ do anos. Talvez por isso os cuidadores de dosos mais velhos estejam men sa feitos com o ambiente físico, condiçõ , de mora lia, recursos financeiros, serviços de sa de e tran porte, itens relativos ao dom' $11 \mathrm{O}$ Meio . ente. A idade do cuidador par e ser.$m$ rariável importante na avaliação ta c alidad de vida deste. ${ }^{15}$ No present estuc quanto maior a idade do cuid $\mathrm{A}_{\text {a }}$ menor escore no domínio Físico. Fm estudo om cuidadores de pacientes com esqu zofrenia, ${ }^{b}$ constatou-se que quanto maior a : ade, maior a sobrecarga percebida do c. dador. Cuidadores mais velhos parecem mais cuscuptíveis a sobrecarga, porém os mais jovens podem sofrer mais isolamento e maiores restrições sociais, proporcionais às maiores possibilidades de atividades de lazer e sociais de sua faixa etária. ${ }^{13}$ Foi encontrada correlação positiva entre o número de pessoas que moram no mesmo domicílio e o domínio Relações Sociais. O número de pessoas que residem no mesmo domicílio parece significar também uma melhor rede de apoio, visto que há mais pessoas próximas que podem ajudar e se revezar no cuidado.

Os cuidadores que referiram maior sobrecarga avaliaram menos satisfatoriamente sua saúde qualidade de vida, o que demonstra que a aplicação de um instrumento específico para s atividades do cuidador correlac ono. se satisfatoriamente com o instrumento a avaliaçà subjetiva de qualidade de vida \& ral, WHOQOL-bref. Além disso, nu nu st. am bom indicador da situaçãe '̀o cul ${ }^{\prime} r^{\prime}$ or, pudendo fornecer subsídios par , llanejan nto de ações e intervenções destinadas àq les que apresentam maior sobrecarg - 'ie, portanı, têm a QV mais comprometida. Por ${ }^{\circ}{ }^{+}$, verificou-se que indivídur n is so recarregados pela tarefa de cuidar tprese na minuição na percepção de sur qual ' de de vida.

F. resença de um companheiro, que pode ser o próp $>$ idoso ou não, pode trazer benefícios com relação ao menor número de sentimentos de sc' dão e maior apoio durante o processo de cuidado, pois reduz o sentimento de desamparo, aspecto muito comum nesta atividade. Muitos são os relatos de companheiros que deixaram suas esposas ao ter de receber em suas casas pais ou parentes das mesmas, que já não podiam viver sozinhos e precisaram então recorrer às casas de seus filhos ou outros parentes. Nesta amostra, a presença de companheiro pareceu favorável ao bem-estar dos cuidadores, o que necessariamente pode não ocorrer ou até ocorrer o contrário ou seja, a presença do companheiro pode ser um fator estressor para o cuidador.

A doença também se apresentou como preditor para menor satisfação com a saúde e a QV geral do cuidador. Algumas doenças provocam uma série de sinais e sintomas que podem interferir na avaliação do cuidador de sua vida e saúde. Concomitantemente a propostas de intervenções voltadas para a saúde dos cuidadores, as equipes de saúde devem avaliar o grau de implicação das enfermidades existentes e como essas podem interferir na tarefa de cuidar e na sua qualidade de vida. 


\section{CONCLUSÃO}

O estudo indicou que esses cuidadores domiciliares de idosos estão cuidando de pessoas com moderado grau de incapacidade funcional e que a sobrecarga afeta diretamente a QV desses cuidadores. Somando-se a isso, trata-se de uma população carente social e economicamente, com pouca oferta de área de lazer e cultura, com baixo nível de escolaridade, acometidas por doenças crônicas e que contam quase que, exclusivamente, com a rede de apoio informal familiar. Considerando este aspecto, fica evidenciado neste estudo que o cuidado domiciliar realizado no âmbito da família pode ser um atributo que mascare a realidade cotidiana do cuidador, sobretudo quando se considera o modelo social de cuidado, tendo a família como principal provedora. No entanto, importante se faz mencionar que, independentemente da relação informal e "nata" que a família tem nesse processo, há que se dizer que o sofrimento e o cansaço são variáveis afeitas a este processo.

Além das ações em âmbito particular pu tuaı como orientações de como realizar o cuid assistência à saúde, apoio físico e emo ' ala aos cuidadores, cabem às equipes de saúde $\mathrm{da} \perp$ nília ações que contemplem os $\mathrm{p}$ ncípio fundamentais do Sistema Único de Saú e, possibi tando uma intervenção mais efetixa na nudan . do perfil de saúde e doença essa pr , ulaçao. Isso significa atuações interse riais e cl na participação da comunidade, na nlar jarem as ações que

RECREENCIA

sport “o EF. Caracterização das formas de vida e trabalho das cuidadoras familiares do rograma de assistência domiciliária do

Hospital Universitário da Universidade de São Paulo. Dissertação [ Mestrado em enfermagem da saúde]. -Escola de Enfermagem; 2003.

2. Garrido R, Menezes PR. Impacto em cuidadores de idosos com demência em um serviço psicogeriátrico. Rev. Saúd Pública 2004 dez; 38 (6): 835-41.

3. Rodriguez PR. El apoyo informal a las personas mayors en España y la protección social a la busquem melhorar a qualidade de vida das pessoas idosas e seus cuidadores. Para tanto, propõe-se:

- Investir e melhorar a estrutura social com a participação da comunidade ("empoderamento"), com o objetivo de discutir com melhorar a qualidade de vida.

- Identificar os recursos da cor (suporte social) para prop medid. alternativas para os cuidados dom iliário com a possibilidade de $\mathrm{cr}$ t viços ${ }^{\cdot} \cdot \mathrm{co}$, como centros-dia com ? $\mathrm{s}$ vo de melhorar a quali de de vi a de idosos e dos cuidadores.

- Utilizar, a n ma efetiva, os instrumentos de avaliaçá d oc os nos cadernos de At IÇa Bás a do próprio Ministério da aúde a id ntificar os idosos e avaliar seu au de comprometimento, assim como iden icar a possível sobrecarga do uidador.

Neste estudo, foram identificadas algumas varíveis que podem influenciar na avaliação da $\checkmark \checkmark$ dos cuidadores. Os dados obtidos, através de comprovação estatística significativa, dão subsídios para se identificar fatores de risco associados à pior percepção de qualidade de vida. A escala Zarit permitiu evidenciar que a presença de companheiro e a presença de doença na relação do cuidador com aquele que é cuidado podem ser preditores positivos ou negativos para a percepção da qualidade de vida geral e da satisfação com a saúde dos cuidadores.

dependencia: del facilismo a los derechos de ciudadanía. Rev. Esp. Geriatr. Gerontol 2005 dec; 40 (supl. 3): 5-15.

4. Fleck MPA, et al. Aplicação da versão em português do instrumento abreviado de avaliação da qualidade de vida "WHOQOLbref”. Rev Saúd Pública 2000 abr; 34 (2): 178-83.

5. Peñaranda AP, Ortiz LG, Góngora JN. El cuidador primário de familiares con dependência: calidad de vida, apoyo social y salud mental tese [ Doutorado em psicologia médica] - Universidad de Salamanca/Facultad de Medicina; 2006. 
6. Schestatsky P, et al. Quality of life in a brazilian sample of patients with parkinson's disease and their caregivers. Rev. Bras. Psiquiatr. 2006 set; 28 (3): 209-11.

7. Tamaki EM. Qualidade de vida: individual ou coletiva? Ciênc Saúd Colet 2000; 5( 1). p. 20-22.

8. Riberto M, et al. Reprodutibilidade da versão brasileira da medida de Independência funcional. Acta Fisiátr 2001 Abr; 8 (1): 45-52.

9. Scavufca M. Versão brasileira da escala burden literview para avaliação de sobrecarga em cuidadores de indivíduos com doenças mentais. Rev. Bras. Psiquiatr 2002 mar; 24 (1): 12-7.

10. Watanabe HAW, Derntl AM. Cuidadores de idosos: uma experiência em unidade básica de saúde . MundoSaúde 2005 out/dez; 29 (4): 639-44.
11. Garcia MAA, et al. Idosos e cuidadores fragilizados? Mundo Saúde 2005 out/dez; 29 (4): 645-52.

12. Brouwer WBF, et al. Burden of caregiving: evidence of objective burden, subjective burden, and quality of life impacts on informal caregivers of patients with rheumatoid arthriti Arthritis Rheumatism 2004 aug; 51 (4): 570-7.

13. Karsch UMS. Envelhecimento com dependência: revelando cuidadores. 'o Pauı Educ; 1998.

14. Glozman JM. Quality of life megivers. Neuropsychol rev 2004 dec; 14 (): In 96.

15. Urizar CA, Maldonad JG. Bur 1 of care in families of patients $1 \mathrm{t}$. chizoph enia. Quality life Res 2006 May; 15 (4): $>$ ?-24. 